\title{
MONOGENEOS PARÁSITOS DE Prochilodus nigricans (CHARACIFORMES: PROCHILODONTIDAE) PROVENIENTES DEL MEDIO NATURAL Y DE UN ESTANQUE DE CULTIVO EN LA AMAZONÍA PERUANA
}

\author{
Lizeth HIDALGO PIÑA ${ }^{1}$, Anaí Paola Prissilla GONZÁLES FLORES ${ }^{1}$, Emer Gloria PIZANGO PAIMA ${ }^{1}$, \\ Germán Augusto Murrieta MOREY*2 \\ 1 Universidad Nacional de la Amazonía Peruana, Laboratorio de Hidrobiología, Iquitos-Perú. \\ 2 Universidade Federal do Paraná, Laboratorio de Ecología Molecular e Parasitología Evolutiva, Curitiba, Brasil. \\ * Email para correspondencia: germantiss1106@gmail.com
}

\begin{abstract}
RESUMEN
En los últimos años en la Amazonía peruana se ha venido desarrollando la piscicultura de especies nativas de gran potencial, destacándose al "boquichico", Prochilodus nigricans como una de las especies más promisorias. Este pez en cautiverio puede estar sometido a grandes niveles de estrés lo que puede influenciar negativamente en su resistencia contra infecciones parasitarias. A pesar de su enorme importancia económica, poco o casi nada se conoce con respecto a su fauna parasitaria. Por tanto el presente estudio pretende identificar los monogenoideos branquiales que parasitan a juveniles de $P$. nigricans provenientes del medio natural y de estanques de cultivo. Cuarenta branquias de $P$. nigricans provenientes del medio natural y cuarenta provenientes de un estanque de cultivo fueron analizados. Las especies de monogenoideos identificadas fueron: Apedunculata discoidea, Tereancistrum curimba y T. toksonum. Mayor número de indivíduos fue registrado en peces provenientes del médio natural. El presente estúdio registra por primera vez estas tres espécies en $P$. nigricans. La presencia de las mismas espécies de monogenoideos en peces provenientes de ambos ambientes despierta uma alerta a los piscicultores de la región, ya que bajo condiciones inadecuadas de cultivo, proliferaciones masivas de estos parasitos pueden ocurrir, colocando en riesgo la salud y calidad de los peces.
\end{abstract}

PALABRAS CLAVE: boquichico, branquias, Iquitos, Monogenoidea.

\section{MONOGENEOUS PARASITES OF Prochilodus nigricans (CHARACIFORMES: PROCHILODONTIDAE) ARISING FROM NATURAL ENVIRONMENT AND A CULTIVATION POND IN PERUVIAN AMAZON}

\begin{abstract}
In recent years, the Peruvian Amazon has been developing the fish culture of native species of great potential, standing out the "boquichico", Prochilodus nigricans as one of the most promising species. This fish in captivity can be subjected to high levels of stress which can negatively influence its resistance against parasitic infections. Despite its enormous economic importance, little or almost nothing is known about its parasitic fauna. Therefore, the present study aims to identify the branchial monogenoideans parasitizing young specimens of $P$. nigricans from natural environment and a culture pond. Forty gills of $P$. nigricans from the natural environment and forty from a culture pond were analyzed. The identified monogenoideans were: Apedunculata discoidea, Tereancistrum curimba and T. toksonum. The higher number of individuals were recorded in fish from natural environment. The present study records for the first time these three parasite species in P. nigricans. The presence of the same monogenoideans in fish from both environments arouses alert to the fish farmers of the region, since under inadequate conditions of cultivation, massive proliferations of these parasites can occur, placing the health and quality of the fish at risk.
\end{abstract}

KEYWORDS: boquichico, gills, Iquitos, Monogenoidea. 


\section{INTRODUCCIÓN}

En los últimos años en la Amazonía peruana se ha venido desarrollando la piscicultura de especies nativas de gran potencial, obteniendo resultados promisorios y constituyendo un recurso importante desde el punto de vista nutritivo y socio económico (Tello y Bayley 2001). Entre los peces amazónicos el "boquichico", Prochilodus nigricans Spix \& Agassiz, 1829 está entre las cinco especies que sostienen la pesquería comercial en la región Loreto (Tello y Bayley 2001).

Prochilodus nigricans es un pez de forma alargado que alcanza en su ambiente natural, hasta $50 \mathrm{~cm}$ de longitud y puede llegar a pesar $3 \mathrm{~kg}$ (Flores et al. 2000). Tiene régimen alimenticio detritívoro, alimentándose de residuos orgánicos principalmente algas y pequeños invertebrados que se encuentran adheridas a la superficie de piedras y troncos (Flores et al.2000). Forma grandes cardúmenes y migran en épocas de aguas bajas, para alimentación, y al inicio del periodo de aguas altas con fines reproductivos (Flores et al. 2000).

Los peces en cautiverio están sometidos a grandes niveles de estrés resultantes de la captura, transporte, manipuleo, alta densidad, calidad del agua con exceso de compuestos tóxicos, baja cantidad de oxígeno, $\mathrm{pH}$, temperatura con grandes variaciones y alimentación inadecuada, estos factores alteran la homeostasis del pez, tornándole más sensible y menos resistentes a los parásitos. De esta manera, el equilibrio que existía entre el ambiente, el pez y los parásitos es quebrado, surgiendo epizootias que son de difícil control y normalmente llevan a los peces a la muerte (Malta et al.2001).

En piscicultura, los monogenoideos branquiales se destacan entre los parásitos que pueden ser perjudiciales para los peces cultivados, causando diversos daños como: lesiones y hemorragias en las branquias, disminución de la fertilidad, retardo en el crecimiento, pérdida de peso y en casos severos pueden causar altas mortalidades (Flores-Crespo y Flores 2003).

Estudios sobre la diversidad de especies, sistemática y biología de helmintos presentes en peces de cultivo y ambientes naturales colectados en la región Loreto, Perú, han mostrado que el conocimiento actual de la fauna de parásitos existentes en peces en esta región es escaso, con posibles consecuencias negativas para el desarrollo de la piscicultura. Por tanto el presente estudio pretende identificar los monogenoideos branquiales que parasitan a juveniles de $P$. nigricans provenientes del medio natural y de estanques de cultivo, analizando sus diferentes índices parasitarios para ambos ambientes y comparando los niveles de parasitismo tanto de los peces provenientes del medio natural como los de estanque de cultivo.

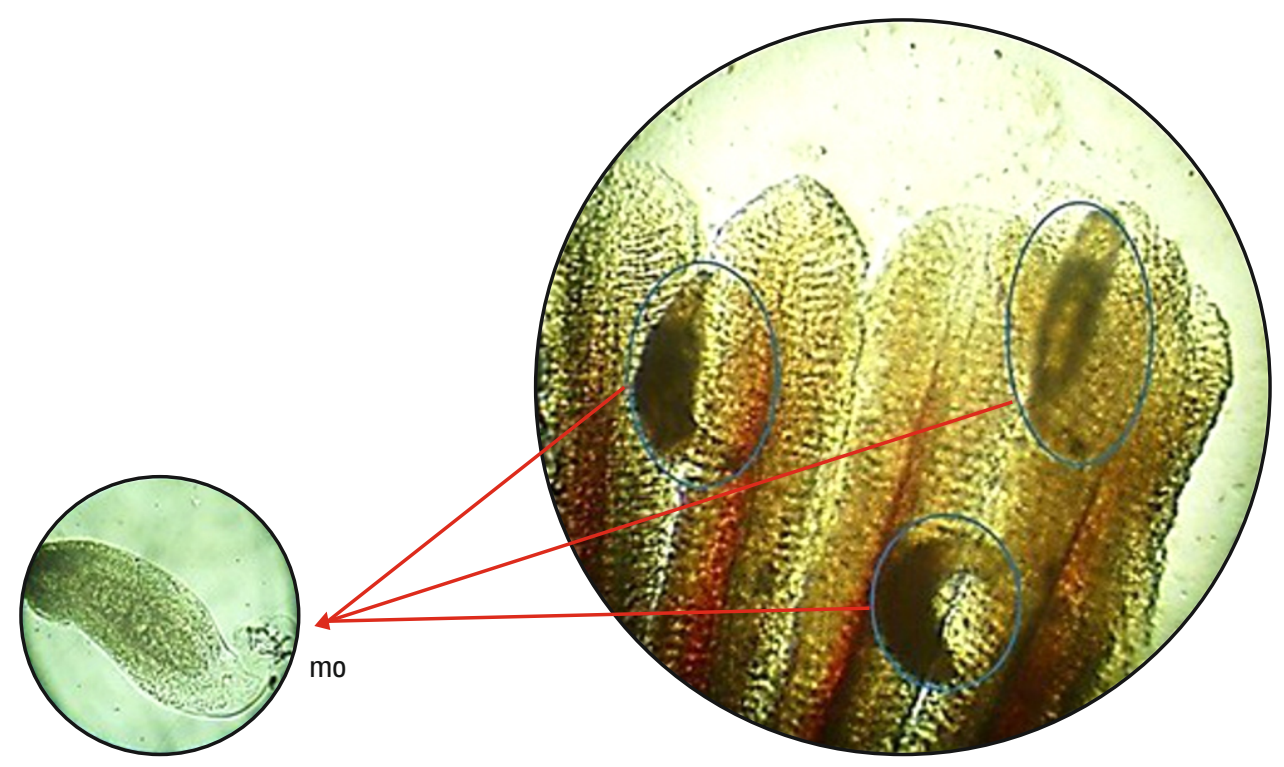

Figura 1. Monogenoideos adheridos a los filamentos branquiales de Prochilodus nigricans Spix \& Agassiz, 1829. mo: monogenoideos 

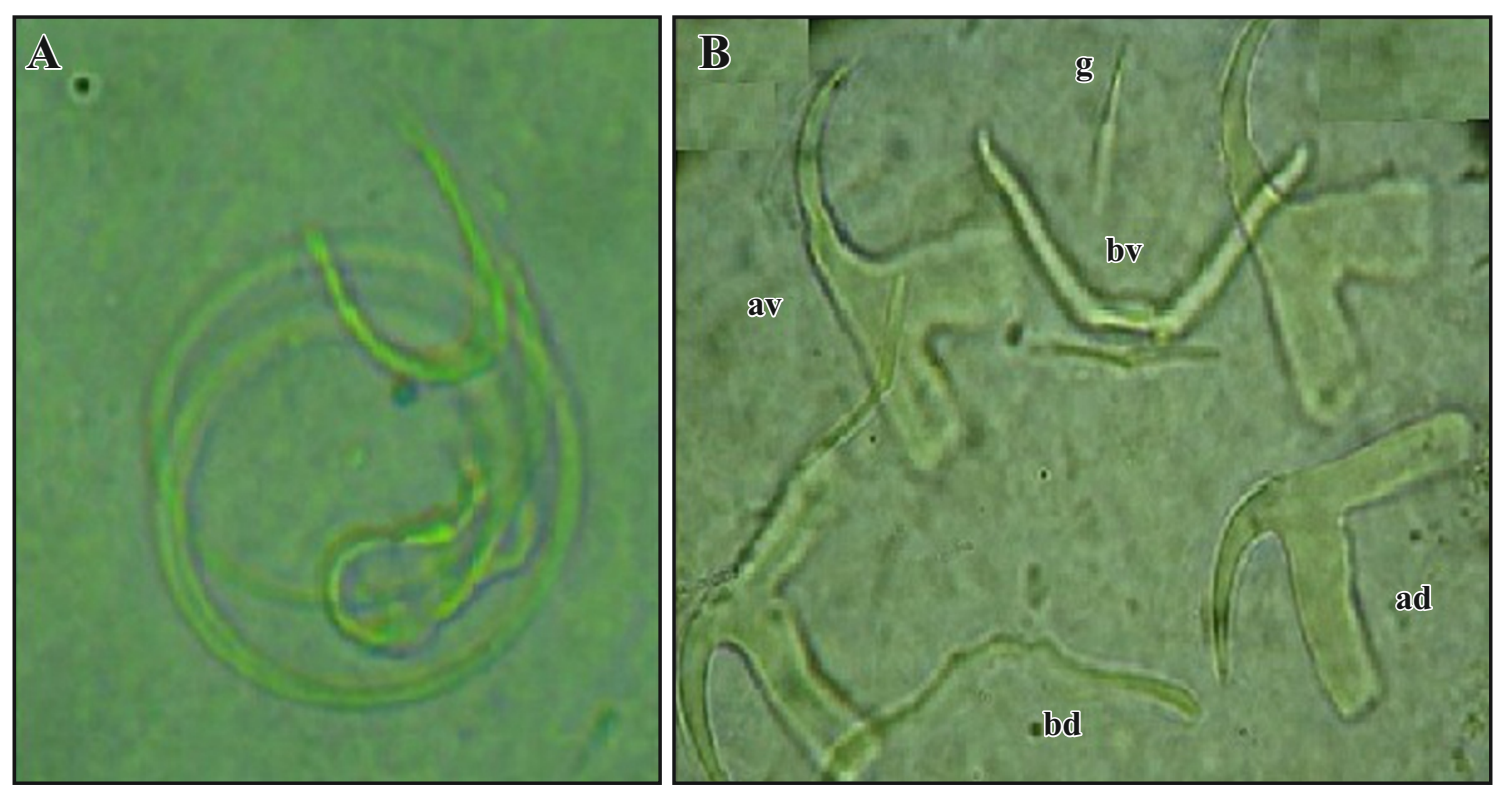

Figura 2. Principales estructuras esclerotizadas de Apedunculata discoidea Cuglianna, Cordeiro \& Luque, 2009. A. Órgano copulador masculino. B. Estructuras de fijación del háptor. av: ancla ventral, bv: barra ventral, ad: ancla dorsal, bd: barra dorsal, g: ganchos

\section{MATERIAL Y MÉTODOS}

El presente estudio se realizó entre los meses de enero hasta abril del 2016, con peces obtenidos del río Amazonas de la comunidad Padre Isla, ubicada entre las coordenadas geográficas $3^{\circ} 41^{\prime} 00.2^{\prime} \mathrm{S}$ y $73^{\circ} 12^{\prime} 12.5^{\prime}$ W, Distrito de Punchana, Provincia de Maynas, Región Loreto y peces obtenidos de estanque de cultivo del Centro de Investigación Experimental y Enseñanza - Piscigranja Quistococha, Facultad de Ciencias Biológicas de la Universidad Nacional de la Amazonía Peruana (CIEE - FCB - UNAP), ubicada entre las coordenadas geográficas $3^{\circ} 49^{\prime} 29.0^{\prime \prime} \mathrm{S}$ y $73^{\circ} 19^{\prime}$ 23.6" W, en el km 4.5 de la Carretera Iquitos - Nauta de la Ciudad de Iquitos, Distrito de San Juan Bautista, Provincia de Maynas, Región Loreto.

Fueron analizados 40 juveniles de $P$. nigricans provenientes del medio natural con $4.71 \pm 1.65$ de longitud estándar, $3.04 \pm 2.14$ de peso y 40 juveniles provenientes de un estanque de cultivo con $5.19 \pm$ 1.40 de longitud estándar y $3.92 \pm 3.21$ de peso.

Los peces fueron sacrificados mediante una perforación en la parte superior de la cabeza con un estilete, realizando un ligero movimiento lateral, provocando inmediatamente la muerte del espécimen, posteriormente fueron tomadas las medidas correspondientes al tamaño y peso de los peces con ayuda de un ictiómetro y una balanza digital.

El análisis de las branquias consistió en retirar los arcos branquiales para ser colocados en placas Petri con agua destilada, siendo observado cada arco branquial por separado en estereoscopio y microscopio (Figura 1). Los parásitos encontrados fueron retirados con ayuda de estiletes y fueron conservados en tubos Eppendorf con formol 5\% para su posterior identificación (Amato et al.2001).

Para la identificación de las especies, fueron confeccionadas láminas con una mezcla de Picrato de Amonio y glicerina (Malmberg 1957) para facilitar la visualización de las estructuras esclerotizadas. Posteriormente, los parásitos fueron observados en microscopio. Las especies fueron identificadas con ayuda de literatura especializada (Cohen y Kohn 2013).

Los índices parasitarios fueron calculados de acuerdo con Bush et al. (1997). Para determinar la diferencia entre el número de monogenoideos de los $P$. nigricans provenientes del medio natural y de estanque de cultivo fue utilizada la prueba no paramétrica de Mann-Whitney, utilizando el paquete estadístico BioEstat 5.0. 


\section{RESULTADOS}

Fueron identificados 3152 monogenoideos, de los cuales 2453 eran de peces provenientes del medio natural y 699 de peces provenientes de un estanque de cultivo. Las especies identificadas fueron: Apedunculata discoidea Cuglianna, Cordeiro \& Luque, 2009 (Figura 2), Tereancistrum curimba Lizama, Takemoto \& Pavanelli, 2004 (Figura 3) y T. toksonum Lizama, Takemoto \& Pavanelli, 2004 (Figura 4).

Los índices parasitarios mostraron a T. curimba como la especie con mayores índices parasitarios tanto para peces provenientes del medio natural como para peces provenientes de un estanque de cultivo (Tabla 1 y 2 ).

El número de monogenoideos provenientes de peces del medio natural fue mayor que el de los peces provenientes de un estanque de cultivo $(\mathrm{Z}(\mathrm{U})=5.07$; $\mathrm{p}=<0.0001)($ Figura 5).

\section{DISCUSIÓN}

La oportunidad de colonizar nuevas especies de hospederos está relacionada a la disponibilidad de adecuados hospederos. Sólo hospederos filogenéticamente o ecológicamente relacionados a sus parásitos, los proveerán de las condiciones necesarias para la sobrevivencia y transmisión (Noble et al.1961). Parásitos con ciclo de vida directo como los ectoparásitos, son frecuentemente encontrados en altos números en ambientes lénticos. Este tipo de ambiente es favorable para la transmisión y proliferación de este tipo de parásitos (Flores-Crespo y Flores 2003). Esto puede explicar la abundancia de monogenoideos encontrados en el presente estudio, los cuales estaban distribuidos en tres especies diferentes, y en elevados números para ambos ambientes.

Tereancistrum curimba e T. toksonum fueron citadas por primera vez parasitando las branquias de P. lineatus (Valenciennes, 1837) provenientes del rio Paraná, Brasil (Lizama et al. 2004). Posteriormente Apedunculata discoidea fue descrita de las branquias de $P$. lineatus provenientes de estanques piscícolas de São Paulo, Brasil (Cugliana et al. 2009).

Lizama et al. (2005), analizando las branquias de $P$. lineatus provenientes del rio Paraná identificaron los monogeneos: T. curimba, T. toksonum, Tereancistrum sp. e Anacanthoroides sp.

Anacanthoroides sanctifrancisci Monteiro \& Brasil-Sato 2014 fue descrita parasitando las branquias de $P$. argenteus Spix \& Agassiz, 1829 del rio São Franciso y $A$. discoidea fue redescrita con ejemplares colectados en este mismo hospedero (Monteiro y Brasil-Sato 2014).

El único estudio reportado sobre la fauna parasitaria de $P$. nigricans en la región neotropical fue realizado por Mathews et al. (2013) quienes identificaron al monogenoideos Rhinonastes pseudocapsaloideum Kritsky, Thatcher \& Boeger, 1988 parasitando las branquias y fosas nasales de peces provenientes de un estanque de cultivo de la ciudad de Iquitos, Perú.
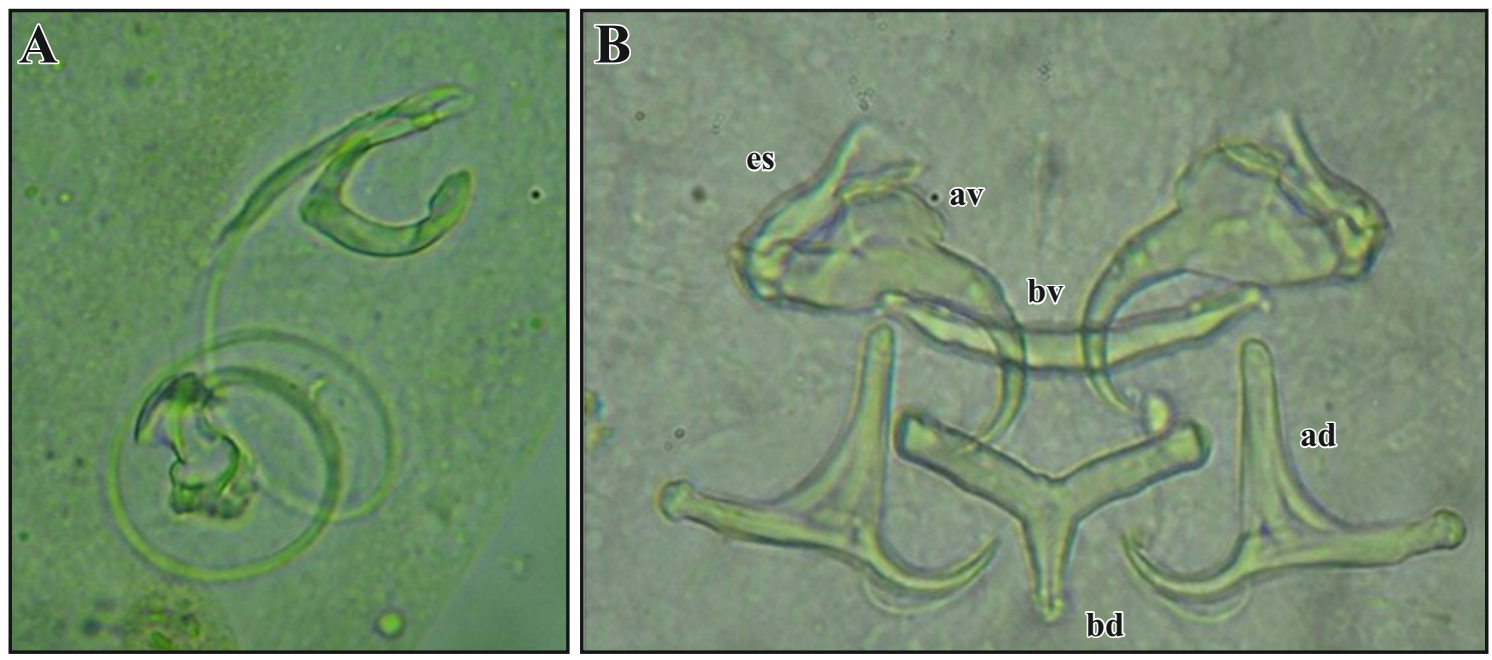

Figura 3. Principales estructuras esclerotizadas de Tereancistrum curimba Lizama, Takemoto \& Pavanelli, 2004. A. Órgano copulador masculino. B. Estructuras de fijación del háptor. av: ancla ventral, bv: barra ventral, ad: ancla dorsal, bd: barra dorsal, es: esclerito 

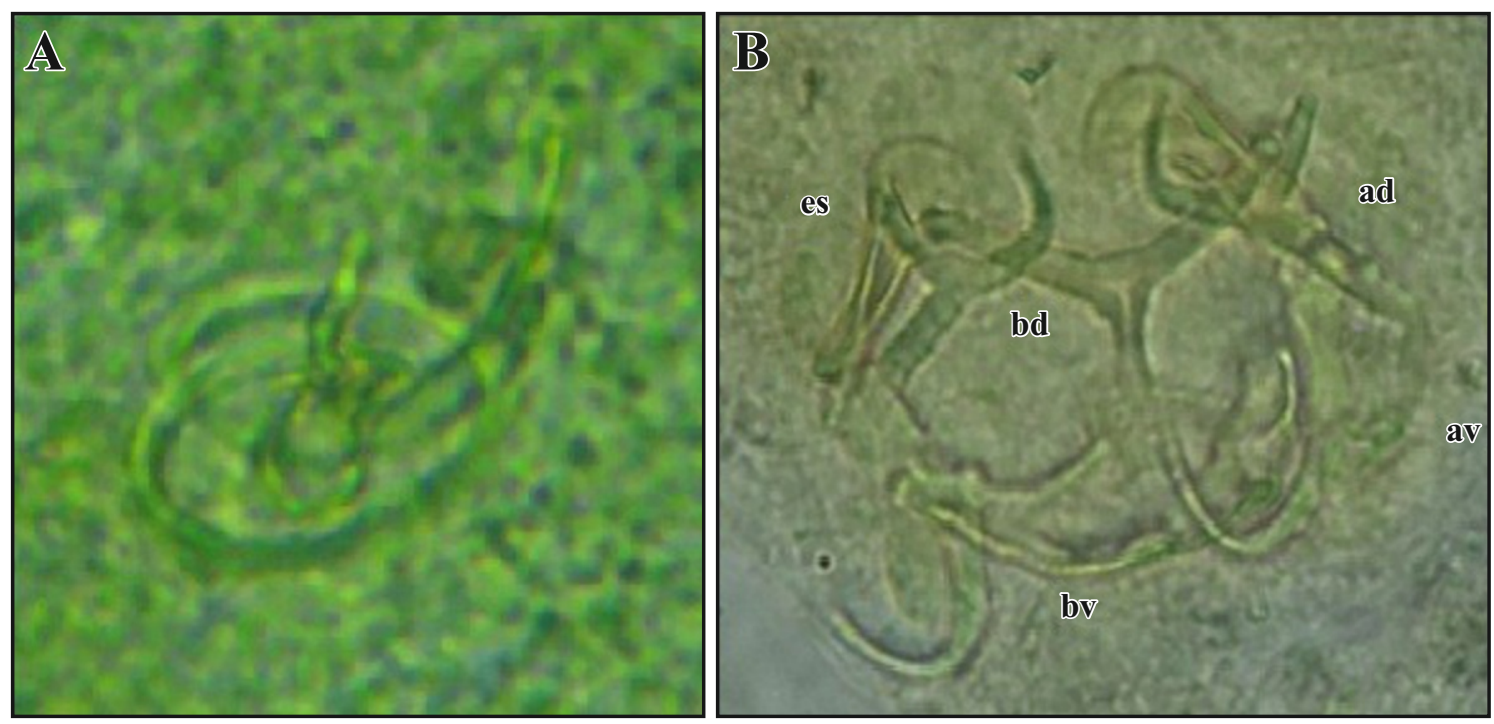

Figura 4. Principales estructuras esclerotizadas de Tereancistrum toksonum Lizama, Takemoto \& Pavanelli, 2004. A. Órgano copulador masculino. B. Estructuras de fijación del háptor. av: ancla ventral, bv: barra ventral, ad: ancla dorsal, bd: barra dorsal, es: esclerito

En el presente estudio, A. discoidea, T. curimba e T. toksonum son registradas por primera vez parasitando $P$. nigricans, constituyendo el primer registro de ocurrencia de estos parásitos en este hospedero. De esta manera, un nuevo hospedero es conocido para estos monogeneos.

Los valores de los índices parasitarios están relacionados a las condiciones inmunológicas del hospedero y a las características genéticas del parásito. Así mismo Flores-Crespo y Flores (2003), mencionan que cuando los peces son sometidos a grandes niveles de estrés resultantes de la captura, transporte, manipuleo, altas densidades, calidad del agua con exceso de compuestos tóxicos, baja cantidad de oxígeno, $\mathrm{pH}$, temperatura con grandes variaciones y alimentación inadecuada, alteran la homeostasis del pez tornándolo más sensible y menos resistente a los patógenos surgiendo infestaciones masivas en los peces de cultivo.

En el presente estudio, los mayores índices parasitarios y mayor cantidad de parásitos fueron reportados para los peces provenientes del medio natural. Esto puede ser consecuencia de las óptimas condiciones de cría en el estanque de cultivo, como: adecuada densidad de siembra, monitoreo constante de los parámetros físico-químicos, buena alimentación de los peces, y cuidado en el manipuleo al momento del muestreo. Todas estas medidas pueden haber contribuido con el bajo estrés de los peces, lo que en consecuencia no alteró sus sistemas inmunes, y aparentemente los dio mayor resistencia a infestaciones parasitarias que los peces provenientes del medio natural.

Al ser los peces comprados de pescadores artesanales, se desconocen las condiciones físicas y químicas de las zonas de pesca, con lo cual no se pueden realizar comparaciones relacionadas a la calidad de agua de ambos ambientes. Probablemente el hábitat natural de los $P$. nigricans utilizados en el presente estudio sea un ambiente más favorable para la colonización de monogeneos branquiales.

La presencia de las mismas especies de monogeneos en los $P$. nigricans provenientes de ambos ambientes sirven de alerta para los piscicultores, ya que los niveles de parasitismo pueden incrementarse considerablemente con la presencia de diversos factores que pueden influenciar negativamente el sistema inmune de los peces, poniendo en riesgo la producción.

Medidas profilácticas son recomendadas desde el inicio de la siembra de los peces en los estanques de cultivo para evitar pérdidas económicas ante algún problema de índole parasitario. 
Tabla 1. Índices parasitários de las espécies de monogeneos parásitos de Prochilodus nigricans Spix \& Agassiz, 1829 provenientes del médio natural.

\begin{tabular}{lcccccc}
\hline \multicolumn{1}{c}{ Espécie } & PE & PP & P\% & I & Im & Am \\
\hline Apedunculata discoidea & 40 & 05 & 12.5 & $28(1-3)$ & 5.60 & 0.70 \\
Tereancistrum curimba & 40 & 40 & 100 & $2393(5-178)$ & 59.83 & 59.83 \\
Tereancistrum toksonum & 40 & 08 & 20 & $32(1-3)$ & 4.00 & 0.80 \\
\hline
\end{tabular}

Tabla 2. Índices parasitários de las espécies de monogeneos parásitos de Prochilodus nigricans Spix \& Agassiz, 1829 provenientes de estanques de cultivo

\begin{tabular}{lcccccc}
\hline \multicolumn{1}{c}{ Espécie } & PE & PP & P\% & I & Im & Am \\
\hline Apedunculata discoidea & 40 & 05 & 12.5 & $16(1-2)$ & 3.2 & 0.4 \\
Tereancistrum curimba & 40 & 40 & 100 & $660(5-81)$ & 16.5 & 16.5 \\
Tereancistrum toksonum & 40 & 05 & 12.5 & $23(1-3)$ & 4.6 & 0.57 \\
\hline
\end{tabular}

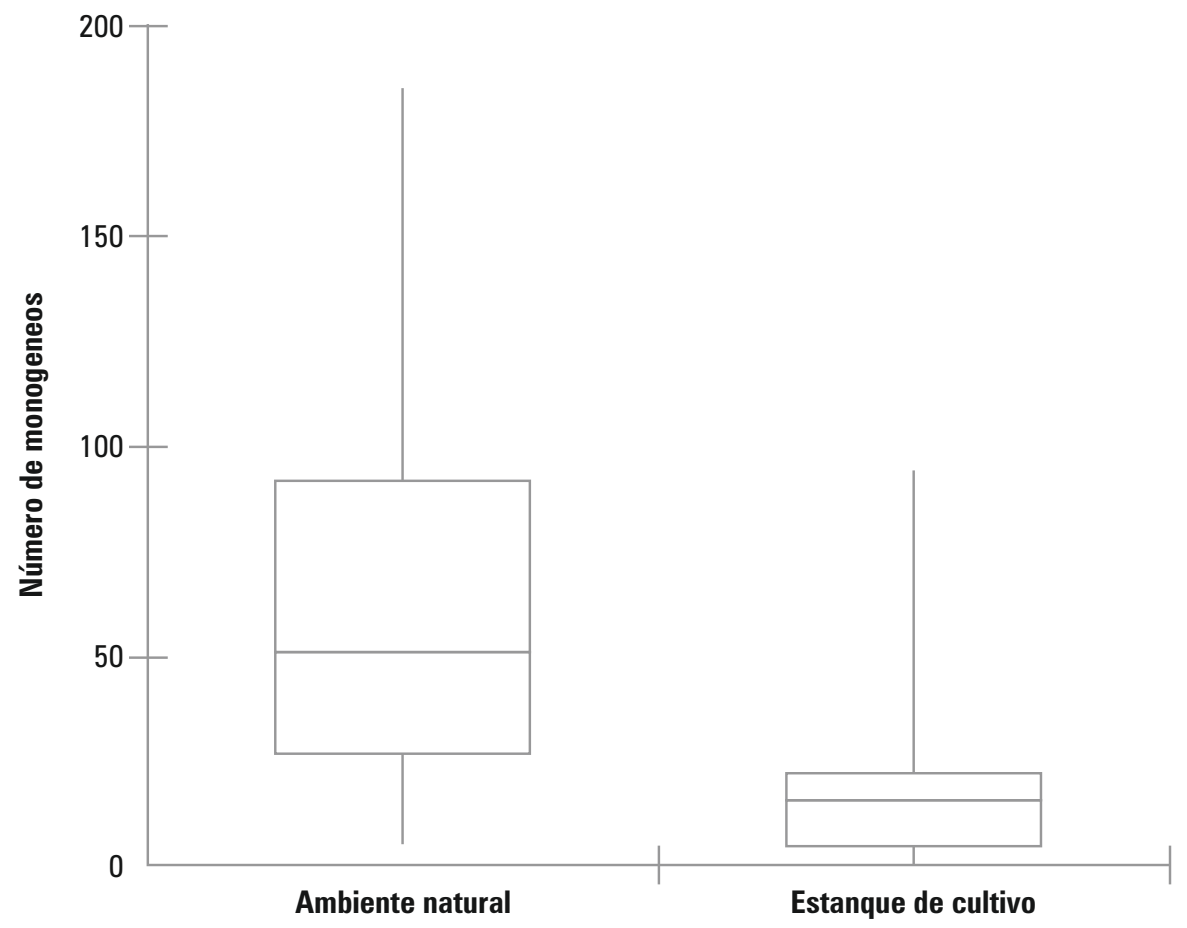

Figura 5. Número de monogenoideos parasitos de Prochilodus nigricans Spix \& Agassiz, 1829 provenientes de estanques del médio natural y de estanque de cultivo. Prueba de Mann-Whitney: $(Z(U)=5.07 ; p=<$ $0.0001)$ 


\section{BIBLIOGRAFIA CITADA}

Amato, J.F.R.; Boeger, W.A.; Amato, S.B. 1991. Protocolos para laboratório-coleta e processamento de parasitas do pescado. Imprensa Universitária, Universidade Federal do Rio de Janeiro. Rio de Janeiro, Brasil. 81pp.

Bush, A.O.; Lafferty, K.D.; Lotz, J.M.; Shostak, A.W. 1997. Parasitology meets ecology on its own terms: Margolis et al. revisited. The Journal of parasitology, 575-583.

Cohen, S.C.; Justo, M.C.; Kohn, A. 2013. South American Monogenoidea parasites of fishes, amphibians and reptiles. Conselho Nacional de Desenvolvimento Cientifico e Tecnologico (CNPq). Oficina de livros, Rio de Janeiro, Brasil. $663 p p$.

Cuglianna, A.M.; Cordeiro, N.S.; Luque, J.L. 2009. Apedunculata discoidea gen. n., sp. n. (Monogenea, Dactylogyridae) parasitic on Prochilodus lineatus (Valenciennes, 1837) (Characiformes: Prochilodontidae) from southeastern Brazil. Brazilian Journal of Biology, 69: 895-898.

Flores, H.; Alcántara, F.; Rebaza, M.; Rebaza, C.; Deza, T.; Tello, S.; Tello, G. 2000. Cultivo y procesamiento de peces nativos: una propuesta productiva para la Amazonía peruana. Repositório del Instituto de Investigaciones de la Amazonía Peruana, Iquitos, Perú. 86pp.

Flores-Crespo, J.F.; Crespo, R.F. 2012. Monogeneos, parásitos de peces en México: estudio recapitulativo. Revista Mexicana de Ciencias Pecuarias, 41: 175-192

Lizama, A.M.; Takemoto, R.M.; Pavanelli, G. C. 2005. Influence of host sex and age on infracommunities of metazoan parasites of Prochilodus lineatus (Valenciennes, 1836) (Prochilodontidae) of the upper Paraná River floodplain, Brazil. Parasite, 12: 299-304.
Malmberg, G. 1957. On the occurrence of Gyrodactylus on Swedish fishes. Sartryck ur Skrifter utgivna av Sodra Sveriges Fiskerifo"rening Arsskrift, 1956: 19-76

Malta, J.C.; Gomes, A.L.; Andrade, S. M.; Varella, A.M. 2001. Massive infestation by Neoechinorhynchus buttnerae Golvan, 1956 (Eoacanthocephala: Neochinorhynchidae) in Young" Tambaquis" Colossoma macropomum (Cuvier, 1818) cultured in the Central Amazon. Acta Amazonica, 31: 133-143.

Mathews, D.P.; Mathews, J.P.; Ismiño, R.O. 2013. Parasitic infections in juveniles of Prochilodus nigricans kept in a semi-intensive fish farm in the Peruvian Amazon. Bulletin European Association of Fish Pathologists, 33: 28-32.

Monteiro, C.M.; Brasil-Sato, M.C. 2014. A new species of Anacanthoroides and redescription of Apedunculata discoidea (Monogenoidea) parasitizing Prochilodus argenteus (Actinopterygii) from the São Francisco River, Brazil. Zootaxa, 3784:259-266.

Noble, E.R.; Noble, G.A. 1961. Parasitology. The Biology of Animal Parasites. Parasitology. The Biology of Animal Parasites. 3rd ed. Lea \& Febiger, Philadelphia, 644pp.

Tello, S.; Bayley, P. 2001. La pesquería comercial de Loreto con énfasis en el análisis de la relación entre captura y esfuerzo pesquero de la flota comercial de Iquitos, cuenca del Amazonas (Perú). Folia Amazónica, 12: 123-139.

Recibido: 05 de Junio del 2017

Aceptado para publicación: 12 de Julio del 2017 
